\title{
Anodic voltammetry and AFM imaging of picomoles of adriamycin adsorbed onto carbon surfaces
}

\author{
Ana Maria Oliveira-Brett*, José António P. Piedade, Ana-Maria Chiorcea \\ Departamento de Química, Faculdade de Ciências e Tecnologia, Universidade de Coimbra, 3004-535 Coimbra, Portugal \\ Received 14 January 2002; received in revised form 8 April 2002; accepted 9 May 2002
}

\begin{abstract}
Adriamycin adsorbs strongly and irreversibly onto surfaces and this enabled electrochemical detection of in situ adriamycin oxidative damage to DNA. The adsorption of adriamycin onto glassy carbon and highly oriented pyrolytic graphite (HOPG) electrodes was studied by voltammetry and mode atomic force microscopy (MAC). At a glassy carbon electrode (GCE), the adsorbate has similar voltammetric behaviour to adriamycin in solution, which enabled the cyclic, differential pulse and square wave voltammetric study of the electron transfer reaction. The total surface concentration of adriamycin adsorbed onto GCE, from a 50 $\mathrm{nM}$ adriamycin solution during $3 \mathrm{~min}$, was calculated to be $2.57 \times 10^{-12} \mathrm{~mol} \mathrm{~cm}^{-2}$. The oxidation of adsorbed adriamycin is pHdependent and corresponds to a two electron/two proton mechanism, and the detection limit for adriamycin adsorbed onto the GCE was $3.33 \times 10^{-10} \mathrm{M}$. In situ AFM images show quick and spontaneous adsorption of the adriamycin onto a HOPG surface. Adriamycin forms a stable monolayer when adsorbed from different concentrations of adriamycin solutions and for short adsorption times. The strong and irreversible chemisorption of adriamycin onto carbon electrodes enables detection limits of the order of picomolar, which is much lower than the detection limits attainable by voltammetric methods for most organic compounds. (C) 2002 Elsevier Science B.V. All rights reserved.
\end{abstract}

Keywords: Voltammetry; Adriamycin; DNA-biosensor; Adsorption; AFM; Electrochemistry

\section{Introduction}

Adriamycin is an antibiotic of the family of anthracyclines with a wide spectrum of chemotherapeutic applications and antineoplasic action but causes very high cardiotoxicity that ranges from a delayed and insidious cardiomyopathy to irreversible heart failure [1]. Adriamycin is the commercial name for doxorubicin hydrochloride [5,12-naphthacenedione, 10-[(3-amino-2, 3, 6-trideoxy-alpha-L-lyxo-hexopyranosyl)oxy]-7, 8, 9, 10-tetrahydro-6, 8, 11-trihydroxy-8-(hydroxylacetyl)-1methoxy-, hydrochloride(8S-cis)] (Scheme 1).

Although the antitumour properties of adriamycin have been known for more than 30 years [2], pharmokinetic and biochemical studies to establish its mechanism of action and improve its administration and anticancer activity are still an important goal. There is

* Corresponding author. Tel./fax: +351-239-835295

E-mail address: brett@ci.uc.pt (A.M. Oliveira-Brett). experimental evidence that adriamycin can promote oxidative damage to double-stranded DNA (dsDNA) in cancerous cells through the generation of reactive oxygen species [3-6], that adriamycin and analogous anthracycline compounds interact with dsDNA through intercalation [1,7-10] and that adriamycin actively accumulates in the nuclear genome [11].

Interactions of several substances with dsDNA have been successfully studied using a recently-developed voltammetric DNA-biosensor [12,13]. This DNA-biosensor allowed the clarification of not only the specific interaction of some drugs with a certain DNA base but also of the electrochemical mechanism of the interaction [13].

Adriamycin intercalation and in situ interaction with double helix DNA was also investigated using the voltammetric DNA-biosensor [14]. The results showed that adriamycin can undergo oxidation or reduction after being intercalated into the DNA double helix. A mechanism proposed for adriamycin reduction and oxidation in situ when intercalated in double helix 
$\mathbf{A}$
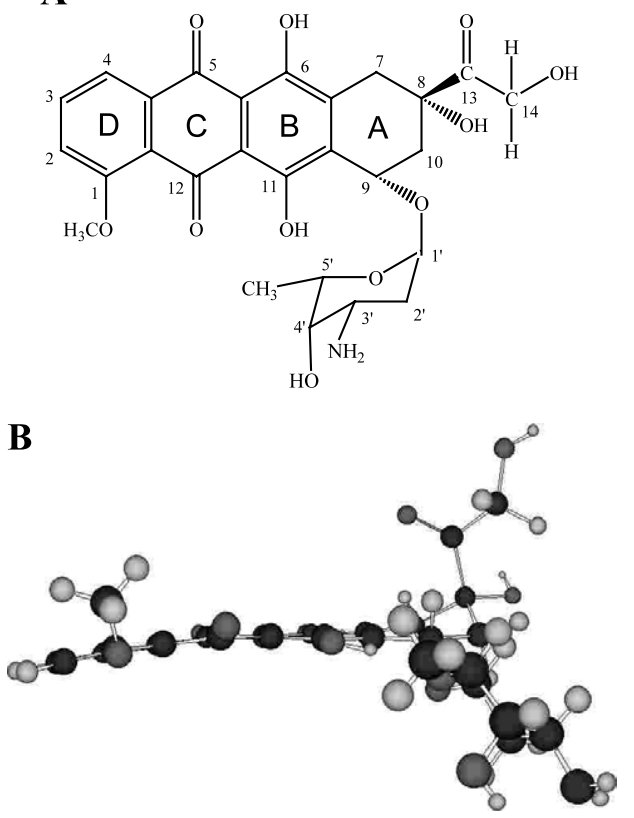

Scheme 1. (A) Structure of adriamycin. (B) 3D representation of adriamycin.

DNA immobilised onto the glassy carbon electrode (GCE) surface showed preferential interaction with the guanine moiety and leads to the formation of the mutagenic 8-oxoguanine [15].

Detection and quantification of adriamycin in organic fluids or cells is thus very important. Electroanalytical methods are inexpensive, fast, and can be automated, and appropriate voltammetric methods can allow direct measurements on biological samples with very little or no sample pre-treatment. This is an enormous advantage when compared with other analytical techniques. Electroanalysis has been used to quantify adriamycin in biological fluids with low detection limits $\left(10^{-8}-10^{-7}\right.$ M) [16-19].

Carbon paste electrodes were used to study the reduction and oxidation of adriamycin [18]. It was found that the oxidation of the 6,11-dihydroquinone functionality is reversible, occurs at $+0.5 \mathrm{~V}$ versus saturated calomel electrode (SCE) (at $\mathrm{pH} 4.5$ ) and is also $\mathrm{pH}$-dependent. Adriamycin adsorbed very strongly and irreversibly onto carbon paste, whilst retaining its characteristic electroactivity in the adsorbed state and this allowed the application of the electroanalytical method to direct determinations in urine samples [19].

This strong and irreversible adsorption of adriamycin on carbon paste electrodes is also observed at GCE and at highly oriented pyrolytic graphite (HOPG) and will be described in the present paper.

Atomic force microscopy (AFM) can bring important information about the adsorption, nucleation and growth of biological compounds at solid surfaces. Magnetic alternating current mode AFM (MAC mode
AFM) is a technique that permits the visualisation of the molecules stacked on the surface by soft interactions [20]. The AFM tip oscillates as it scans the sample, touching the sample surface only at the bottom of the oscillation. MAC mode uses a solenoid placed under the sample to oscillate the magnetically coated AFM tip directly. Because there is no need to drive the cantilever holder, cantilever chip and solution as in tapping mode, the control of the cantilever increases considerably, which enables operation at smaller oscillation amplitudes. The lateral forces are better eliminated so the tip does not push the molecules out from the surface of the HOPG.

This paper is concerned with the study and characterisation of the oxidation mechanism of adriamycin adsorbed onto a GCE surface using cyclic, differential pulse and square wave voltammetry, in buffer supporting electrolytes at different values of $\mathrm{pH}$. The adsorption of adriamycin onto HOPG was investigated by in situ MAC mode AFM.

\section{Experimental}

\subsection{Reagents}

Adriamycin (doxorubicin hydrochloride, $2 \mathrm{mg} \mathrm{ml}^{-1}$ solution) was obtained from Pharma-APS, and used without further purification. Solutions of different concentrations of adriamycin were prepared by direct dilution of the appropriate volume in acetate, phosphate, or borate buffer according to the $\mathrm{pH}$ desired.

In all cases buffer electrolyte solutions of ionic strength $0.1 \mathrm{M}$ were used and were prepared using analytical grade reagents and purified water from a Millipore Milli-Q system (conductivity $<0.1 \mu \mathrm{S} \mathrm{cm}^{-1}$ ).

\subsection{Apparatus}

All voltammetric experiments were done using an MAUTOLAB running with GPES version 4.7 software from Eco-Chemie, Utrecht, The Netherlands. A one compartment electrochemical cell with a volumetric capacity of 5 $\mathrm{ml}$ was used containing a $\operatorname{GCE}(d=6 \mathrm{~mm})$ as the working electrode (WE), a Pt wire counter electrode (CE), and a SCE as the reference.

The experimental conditions unless stated otherwise were: cyclic voltammetry $(\mathrm{CV})$, scan rates varying from $1 \mathrm{mV} \mathrm{s}^{-1}$ to $5 \mathrm{~V} \mathrm{~s}^{-1}$; differential pulse voltammetry pulse, amplitude $50 \mathrm{mV}$, pulse width $70 \mathrm{~ms}$, scan rate 5 $\mathrm{mV} \mathrm{s}^{-1}$; square wave voltammetry, pulse amplitude 50 $\mathrm{mV}$, frequency $50 \mathrm{~Hz}$, corresponding to an effective scan rate of $100 \mathrm{mV} \mathrm{s}^{-1}$.

For dosing nano- and microvolumes EP-10 Plus and EP-100 Plus motorised microliter pippette (Rainin Instrument Co. Inc., Woburn, USA), an Eppendorf 
Research 20-200 (Eppendorf, Hamburg, Germany), and for 500 and $1000 \mu \mathrm{l}$ a Biohit Proline pipette (Helsinki, Finland) were used. The $\mathrm{pH}$ measurements were carried out with a Crison Model micropH 2001 pH-meter with an Ingold combined glass electrode. All experiments were done at room temperature (r.t.).

AFM was performed with a Pico SPM controlled by a MAC Mode module and interfaced with a PicoScan controller from Molecular Imaging Co. Silicon type II MAClevers of $225 \mu \mathrm{m}$ length, $2.8 \mathrm{~N} \mathrm{~m}^{-1}$ spring constants and $27-30 \mathrm{kHz}$ resonant frequencies in liquid (Molecular Imaging Co.) were used. HOPG, grade $\mathrm{ZYH}$, from Advanced Ceramics Co., was used as a substrate. Before experiments the freshly cleaved HOPG substrate was imaged by Contact Mode AFM in order to establish its cleanliness. AFM of the samples was carried out in a one-compartment Teflon cell of approximately $12.5 \mathrm{~mm}$ internal diameter holding the HOPG sample on the base. All images (256 samples per line $\times 256$ lines) were taken at r.t., scan rate 1.95 lines per $\mathrm{s}$. The images were processed by flattening in order to remove the background slope and the contrast and brightness were adjusted.

ORIGIN (version 6.0) from Microcal Software was used for the presentation of all the experimental voltammograms and graphs reported in this work. When needed, the experimental voltammograms were smoothed using a Savitsky-Golay smoothing algorithm.

CHEMDRAW ULTRA and CHEM3D PRO (version 5.0) from Cambridge Soft Corporation were used to draw the structure of the adriamycin molecule. The critical volume and the dimensions of the adriamycin molecule were calculated using CHEMDRAW ULTRA based on the Joback fragmentation method and CHEM3D PRO respectively.

\subsection{Adriamycin adsorbed layer}

The adsorbed layer of adriamycin was prepared by immersing the GCE in solutions of different adriamycin concentrations, to be specified for each experiment, in $\mathrm{pH} 4.50 .1 \mathrm{M}$ acetate buffer electrolyte solution, for 3 min, followed by thorough cleaning of the electrode with a jet of deionised water. The electrode was then transferred to the chosen electrolyte buffer solution and the voltammetric measurements were done immediately.

In the AFM experiments the solutions of adriamycin were placed in the AFM cell and the adriamycin was left to adsorb to the HOPG surface over periods from $30 \mathrm{~s}$ to $3 \mathrm{~min}$. The HOPG with adsorbed adriamycin was cleaned with a jet of Milli-Q water and transferred into $\mathrm{pH}$ 4.5, 0.1 M buffer acetate solution for imaging.

\section{Results and discussion}

\subsection{Anodic voltammetry of adsorbed adriamycin}

Voltammetric experiments performed with the GCE using adriamycin solutions led to great difficulty in cleaning the electrode surface. This indicated a very strong and irreversible adsorption of adriamycin onto the GCE surface. Under the electrochemical conditions used, saturation of the electrode surface can be seen for a concentration of $9.0 \times 10^{-7} \mathrm{M}$ adriamycin in buffer solution (Fig. 1).

In order to investigate the irreversible chemisorption of adriamycin onto the GCE cyclic, differential pulse and square wave voltammetry experiments were performed.

Differential pulse voltammograms obtained in a $1 \mu \mathrm{M}$ adriamycin $\mathrm{pH} \quad 4.5 \quad 0.1 \mathrm{M}$ acetate buffer electrolyte solution and after adsorption as described in the Section 2, are overlaid in Fig. 2. This comparison shows no identifiable differences between the first voltammograms obtained with adriamycin in the bulk solution and those obtained from adriamycin adsorbed on the electrode surface. Similar behaviour was obtained even when adriamycin was adsorbed onto the GCE surface after deposition of $3 \mathrm{~min}$ from $\mathrm{pH} 4.50 .1 \mathrm{M}$ acetate buffer electrolyte adriamycin solutions with lower concentrations of $10^{-10}-10^{-9} \mathrm{M}$ (Fig. 9).

The same experiment of Fig. 2 was performed using square wave voltammetry (Fig. 3), and no appreciable decrease on the first scan for the net current curve of the adsorbed layer of adriamycin was observed. Moreover, the identical value for the peak potential (Fig. 3(a)), for both forward and backward currents is an clear indication that charge transfer is mostly due to adsorbed adriamycin onto the GCE surface [21]. From this square wave voltammogram the reversible character of the electron transfer oxidation reaction of adriamycin can

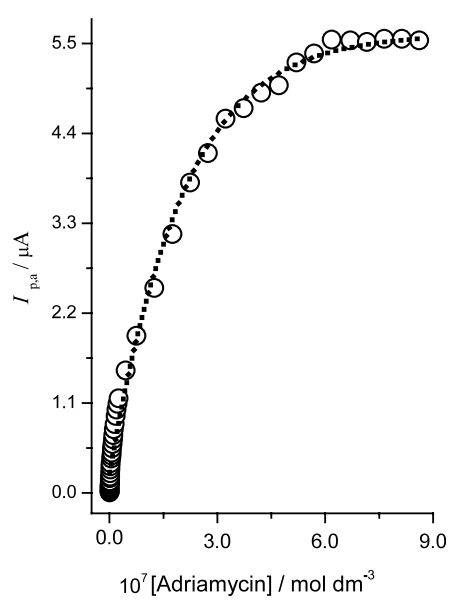

Fig. 1. Differential pulse voltammetric peak current vs. adriamycin concentration in $\mathrm{pH} 4.50 .1 \mathrm{M}$ acetate buffer. 


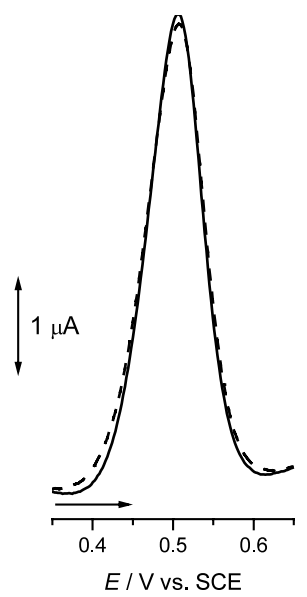

Fig. 2. Differential pulse voltammograms in $\mathrm{pH} 4.50 .1 \mathrm{M}$ acetate buffer electrolyte, at a GCE: (-) $1 \mu \mathrm{M}$ adriamycin solution, and (---) adriamycin adsorbed onto GCE, after $3 \mathrm{~min}$ free adsorption from a $\mathrm{pH}$ $4.50 .1 \mathrm{M}$ acetate buffer electrolyte $1 \mu \mathrm{M}$ adriamycin solution and transferred to buffer after rinsing the electrode with deionised water.
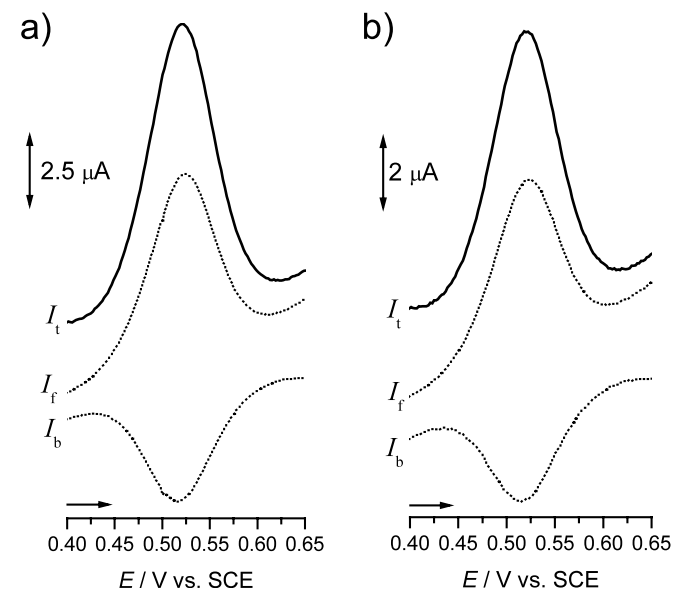

Fig. 3. Square wave voltammograms in $\mathrm{pH} 4.50 .1 \mathrm{M}$ acetate buffer electrolyte, at a GCE: (a) $1 \mu \mathrm{M}$ adriamycin solution; (b) adriamycin adsorbed onto the GCE, after deposition of $3 \mathrm{~min}$ from a pH $4.50 .1 \mathrm{M}$ acetate buffer electrolyte $1 \mu \mathrm{M}$ adriamycin solution, and transferred to buffer after rinsing the electrode with deionised water.

be seen, in bulk solution as well as on the adsorbed layer. This shows that adriamycin adsorbed very strongly and irreversibly onto glassy carbon, whilst retaining its characteristic electroactivity in the adsorbed state.

\subsection{Effect of $p H$ on oxidation of adsorbed adriamycin}

The peak current for the oxidation of adsorbed adriamycin onto the GCE is strongly dependent on the $\mathrm{pH}$ of the supporting electrolyte. Consecutive scans in $\mathrm{pH} 4.5$ acetate buffer electrolyte solution, using differential pulse voltammetry (Fig. 4), showed a continuous, but slow, decrease in the peak current. However, a much more rapid decrease of the adriamycin oxidation peak current was observed when the electrode with the

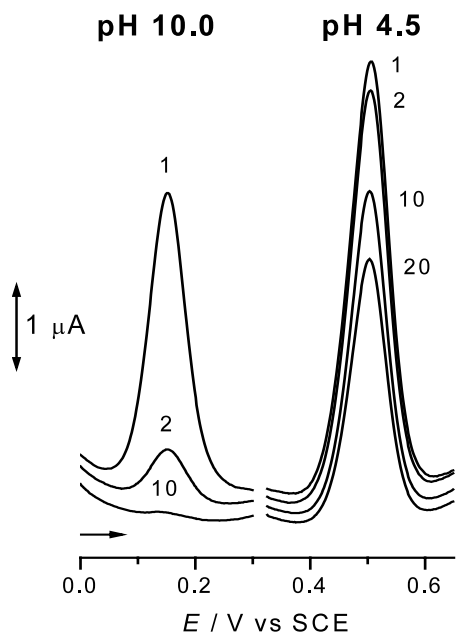

Fig. 4. Successive differential pulse voltammograms in $\mathrm{pH} 4.50 .1 \mathrm{M}$ acetate buffer electrolyte solution (scans 1, 2, 10 and 20), and in $\mathrm{pH}$ $10.00 .1 \mathrm{M}$ borate buffer electrolyte solution (scans 1, 2 and 10) of adriamycin adsorbed, after deposition of 3 min from a pH $4.50 .1 \mathrm{M}$ acetate buffer electrolyte $1 \mu \mathrm{M}$ adriamycin solution, onto the GCE.

adsorbed layer was immersed in a $\mathrm{pH} 10.0$ borate buffer electrolyte solution (Fig. 4). This is due to desorption of adsorbed adriamycin in alkaline solution. Values for the oxidation peak currents, of adsorbed adriamycin, for successive scans at $\mathrm{pH} 4.5$ and 10.0 are given in Table 1 in which the values are compared with $I_{\mathrm{p}, \mathrm{a}}=5 \mu \mathrm{A}$, the value obtained for the peak current for $1 \mu \mathrm{M}$ adriamycin in $\mathrm{pH} 4.50 .1 \mathrm{M}$ acetate buffer solution. Immersing the electrode in $\mathrm{pH} 10.0$ was found to be the best procedure to clean the electrode.

The effect of $\mathrm{pH}$ on the oxidation peak potential, $E_{\mathrm{p}, \mathrm{a}}$, of adriamycin adsorbed onto the GCE surface was studied in appropriate $0.1 \mathrm{M}$ buffer supporting electrolytes, for a wide range of $\mathrm{pH}$ values (Fig. 5). Adsorption of adriamycin onto the GCE surface was carried out for 3 min from a $300 \mathrm{nM}$ adriamycin solution in $0.1 \mathrm{M} \mathrm{pH}$ 4.5 acetate buffer electrolyte. After adsorption the electrode surface was thoroughly washed with deionised water and the voltammetric measurement for each $\mathrm{pH}$ was done immediately. The oxidation of adriamycin is $\mathrm{pH}$ dependent and since the dashed line (Fig. 5(a)),

Table 1

Values of $I_{\mathrm{p}, \mathrm{a}}$ for successive differential pulse scans at $\mathrm{pH} 4.5$ and 10.0, of adriamycin adsorbed onto $\mathrm{GCE}^{\mathrm{a}}$

\begin{tabular}{lllll}
\hline Scan number & $\mathrm{pH} 4.5$ & & $\mathrm{pH} 10.0$ \\
& $I_{\mathrm{p}, \mathrm{a}} / \mu \mathrm{A}$ & $\% I^{\mathrm{b}}$ & $\begin{array}{l}I_{\mathrm{p}, \mathrm{a}} / \mu \mathrm{A} \\
\% I^{\mathrm{b}}\end{array}$ \\
\hline 1 & 5 & 100 & 3.46 & 69.2 \\
2 & 4.86 & 97.2 & 0.55 & 11 \\
10 & 3.81 & 76.2 & 0.04 & 0.8 \\
\hline
\end{tabular}

a Adriamycin was adsorbed onto GCE from a $1 \mu \mathrm{M}$ adriamycin in $\mathrm{pH} 4.50 .1 \mathrm{M}$ acetate buffer solution were the peak current was $5 \mu \mathrm{A}$. Adsorption time $3 \mathrm{~min}$.

b $\% I=I_{\mathrm{p}, \mathrm{a}} / 5 \mu \mathrm{A}$. 

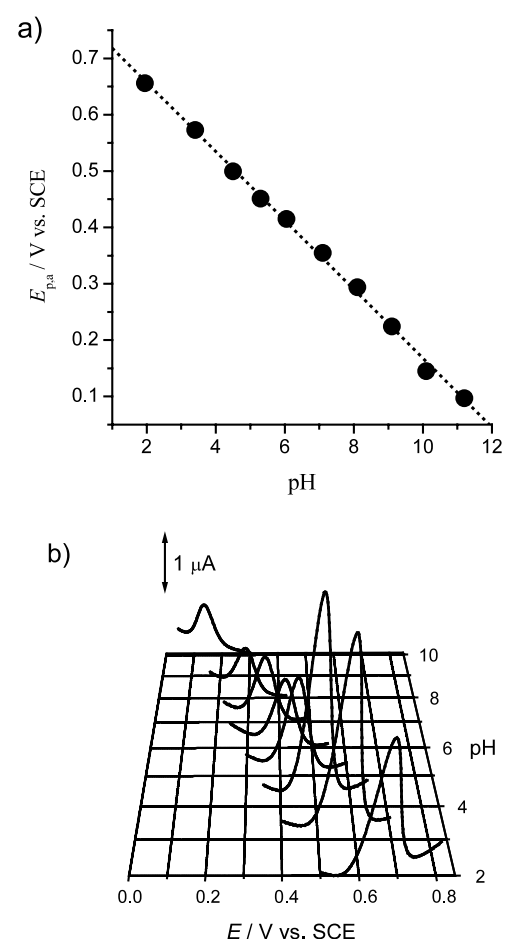

Fig. 5. Differential pulse voltammograms for each $\mathrm{pH}$ were done immediately after washing the electrode surface with deionised water: (a) Plot of $E_{\mathrm{p}}$ vs. pH of adriamycin adsorbed, after deposition of $3 \mathrm{~min}$ from a $\mathrm{pH} 4.50 .1 \mathrm{M}$ acetate buffer electrolyte $1 \mu \mathrm{M}$ adriamycin solution, onto the GCE surface. The dotted line corresponds to a slope of $61 \mathrm{mV}$ per unit of $\mathrm{pH}$. (b) 3D plot of differential pulse voltammograms as a function of $\mathrm{pH}$.

corresponds to $61 \mathrm{mV}$ per unit of $\mathrm{pH}$, the oxidation of adsorbed adriamycin involves the same number of electrons and protons.

In Fig. 5(b) are differential pulse voltammograms of adsorbed adriamycin obtained for different $\mathrm{pH}$ values. The value for $I_{\mathrm{p}, \mathrm{a}}$ obtained in $\mathrm{pH} 4.50 .1 \mathrm{M}$ acetate buffer electrolyte solution is the highest and desorption was slower as is shown in Fig. 4. It was considered that the total amount of adsorbed adriamycin remained constant during the voltammetric measurements in $\mathrm{pH}$ 4.5 acetate buffer and so these conditions were used in all subsequent experiments.

\subsection{Characterisation of the electrode process of adsorbed adriamycin}

Cyclic voltammograms recorded for adriamycin adsorbed onto GCE for scan rates, $v$, between $1 \mathrm{mV} \mathrm{s}^{-1}$ and $4 \mathrm{~V} \mathrm{~s}^{-1}$, are presented in Figs. 6 and 7. Before recording each cyclic voltammogram the electrode was immersed for $3 \mathrm{~min}$ in a $50 \mathrm{nM}$ adriamycin solution, rinsed with deionised water, and placed in $\mathrm{pH} 4.50 .1 \mathrm{M}$ acetate buffer where the cyclic voltammetry was done. The cyclic voltammograms obtained for $1 \leq v \leq 10 \mathrm{mV}$ $\mathrm{s}^{-1}$ presented symmetrical wave shapes, with no significant or very small separation between anodic and

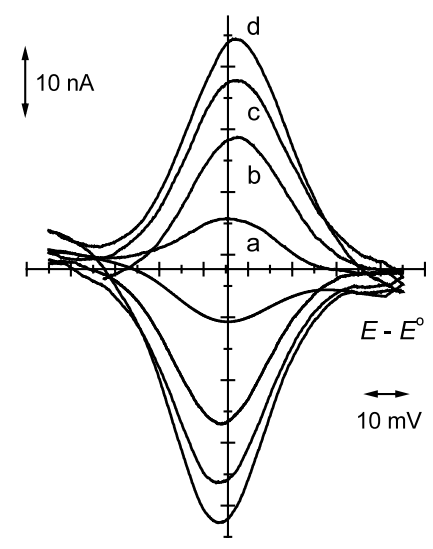

Fig. 6. Background-subtracted cyclic voltammograms in $\mathrm{pH} 4.50 .1 \mathrm{M}$ acetate buffer electrolyte solution of adriamycin adsorbed, after deposition of $3 \mathrm{~min}$ from a $\mathrm{pH} 4.50 .1 \mathrm{M}$ acetate buffer electrolyte $50 \mathrm{nM}$ adriamycin solution, onto a GCE surface: (a) $1 \mathrm{mV} \mathrm{s}^{-1}$; (b) 3 $\mathrm{mV} \mathrm{s}^{-1}$; (c) $4 \mathrm{mV} \mathrm{s}^{-1}$; (d) $5 \mathrm{mV} \mathrm{s}^{-1}$.

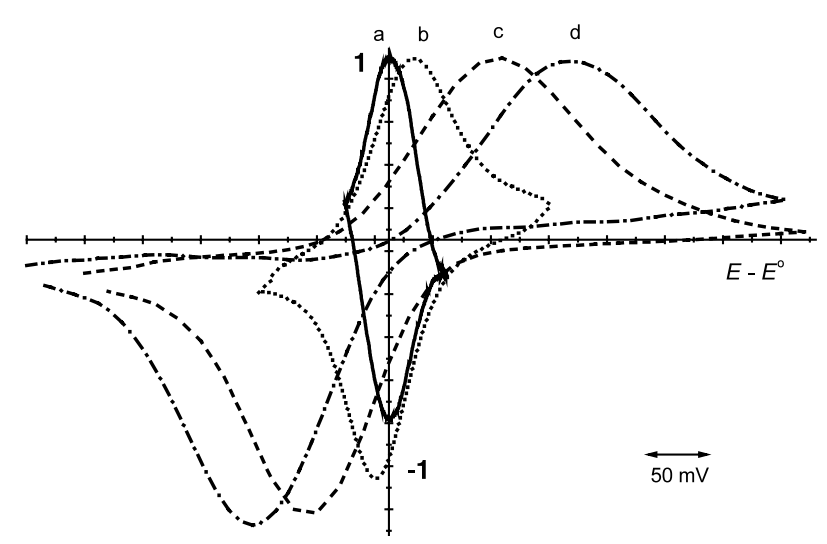

Fig. 7. Background-subtracted cyclic voltammograms in $\mathrm{pH} 4.50 .1 \mathrm{M}$ acetate buffer electrolyte solution of adriamycin adsorbed, after deposition of $3 \mathrm{~min}$ from a $\mathrm{pH} 4.50 .1 \mathrm{M}$ acetate buffer electrolyte $50 \mathrm{nM}$ adriamycin solution, onto a GCE surface: (a) $1 \mathrm{mV} \mathrm{s}^{-1}, 1$ unit $=4 \mathrm{nA}$; (b) $50 \mathrm{mV} \mathrm{s}^{-1}, 1$ unit $=16.7 \mathrm{nA}$; (c) $1 \mathrm{~V} \mathrm{~s}^{-1}, 1$ unit $=1.67$ $\mu \mathrm{A}$; (d) $4 \mathrm{~V} \mathrm{~s}^{-1}, 1$ unit $=5 \mu \mathrm{A}$.

cathodic peak potentials (Table 2). The symmetry of the wave shape for the current-potential curve and the linear dependence of $I_{\mathrm{p}, \mathrm{a}}$ with $v$ is characteristic of reversible charge transfer for adsorbed species in which the contribution of solution-resident species is negligible [22-27]-the electrode was in supporting electrolyte alone with no adriamycin in solution. The small increase of the current peak width at half height, $W_{1 / 2}$, and of anodic and cathodic peak separation with the scan rate (Table 2) can be attributed to slow charge transfer kinetics [24]. The slight asymmetry between the experimental voltammograms obtained and those predicted by the theory for adsorbed species [25] stems from background supporting electrolyte interactions and from the non-ideal behaviour of adsorbed species.

No appreciable variation of the peak potential and a good linear dependence of $I_{\mathrm{p}, \mathrm{a}}$ with $v$ was observed for 
Table 2

Values of peak currents and potentials obtained from cyclic voltammograms of adriamycin for different scan rates

\begin{tabular}{|c|c|c|c|c|c|c|c|c|}
\hline \multirow[t]{2}{*}{ Scan rate $/ \mathrm{mV} \mathrm{s}^{-1}$} & \multicolumn{4}{|c|}{ Peak current/nA } & \multicolumn{4}{|c|}{ Peak potential vs. SCE/mV } \\
\hline & $I_{\mathrm{p}, \mathrm{a}}$ & $\Gamma_{\mathrm{adr}} / \mathrm{pmol} \mathrm{cm}^{-2 \mathrm{a}}$ & $I_{\mathrm{p}, \mathrm{c}}$ & $\Gamma_{\mathrm{adr}} / \mathrm{pmol} \mathrm{cm}^{-2 \mathrm{~b}}$ & $E_{\mathrm{p}, \mathrm{a}}$ & $E_{\mathrm{p}, \mathrm{c}}$ & $\Delta E_{\mathrm{p}}$ & $W_{1 / 2}$ \\
\hline 1 & 8.7 & 2.57 & -8.7 & 2.57 & 532 & 532 & 0 & 43 \\
\hline 2 & 17.0 & 2.52 & -17.0 & 2.52 & 534 & 533 & 1 & 45 \\
\hline 3 & 22.4 & 2.27 & -22.4 & 2.27 & 534 & 530 & 4 & 46 \\
\hline 4 & 31.0 & 2.29 & -32.0 & 2.38 & 534 & 529 & 5 & 50 \\
\hline 5 & 38.0 & 2.25 & -42.0 & 2.49 & 535 & 529 & 6 & 52 \\
\hline 6 & 42.9 & 2.12 & -48.5 & 2.39 & 537 & 528 & 9 & 55 \\
\hline 8 & 58.3 & 2.16 & -66.0 & 2.45 & 537 & 524 & 13 & 52 \\
\hline 10 & 74.1 & 2.19 & -81.9 & 2.42 & 535 & 526 & 9 & 51 \\
\hline
\end{tabular}

Adsorption time $3 \mathrm{~min}$.

${ }^{a}$ Calculated from equation 1 using anodic peak currents.

${ }^{\mathrm{b}}$ Calculated from equation 1 using cathodic peak currents.

$1 \mathrm{mV} \mathrm{s}^{-1} \leq v \leq 500 \mathrm{mV} \mathrm{s}^{-1}(R=0.999)$, corresponding to no diffusional limitations [28]. This is shown in the plot of oxidation peak current, $I_{\mathrm{p}, \mathrm{a}}$, for different values of $v$ (Fig. 8(a)).

For high scan rates of $v>500 \mathrm{mV} \mathrm{s}^{-1}$ a greater variation of the peak potential was found and no linear dependence of $I_{\mathrm{p}}$ with $v$ (Figs. 7 and 8(b)). Fig. 8(b) shows the transition from a reversible to an irreversible system on increasing the sweep rate.

This deviation from linearity could also be explained by considering the effect of very high scan rates on the peak current due to uncompensated resistance [23]. The fact that the cathodic peak current increases more than the anodic with increasing scan rate could be attributed to a faster rate for the cathodic process of the quasireversible mechanism. For very high values of scan rate it is found that adjustments of the theory may be necessary $[22,24,25]$.

For the lowest scan rate, $v=1 \mathrm{mV} \mathrm{s}^{-1}, W_{1 / 2}$ is equal to $45 \mathrm{mV}$ so the number of electrons transferred is equal to 2. Considering the slope of the plot of peak potential versus pH (Fig. 5(a)) it can be concluded that two electrons and two protons are exchanged during the
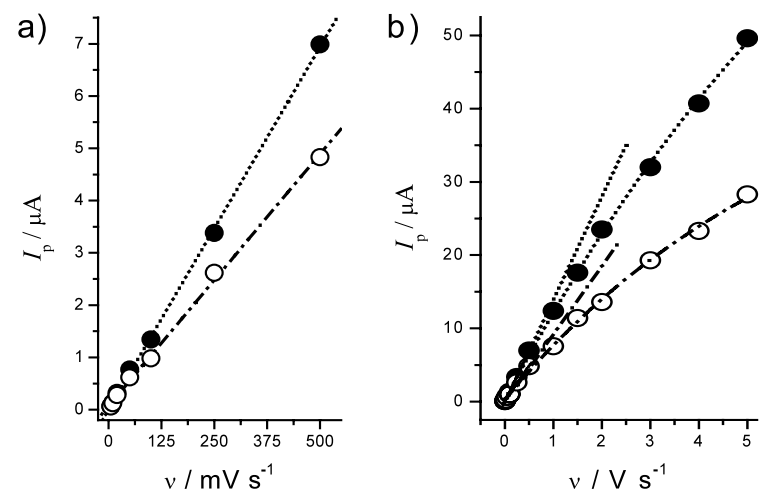

Fig. 8. Cyclic voltammetric peak current in $\mathrm{pH} 4.50 .1 \mathrm{M}$ acetate buffer electrolyte solution of adriamycin adsorbed, after deposition of $3 \mathrm{~min}$ from a $\mathrm{pH} 4.50 .1 \mathrm{M}$ acetate buffer electrolyte $50 \mathrm{nM}$ adriamycin solution, onto a GCE surface vs. scan rate, $(\bigcirc) I_{\mathrm{p}, \mathrm{a}}(\bullet) I_{\mathrm{p}, \mathrm{c}}$. oxidation of adriamycin. The oxidation mechanism deduced is presented in Scheme 2.

\subsection{Calculation of adriamycin concentration on the glassy carbon surface}

As was shown for a very low scan rate of $v=1 \mathrm{mV}$ $\mathrm{s}^{-1}$ (Fig. 6 (curve a)), the wave shapes for the anodic and the cathodic peak are symmetrical, there is no separation between anodic and cathodic peaks, and the ratio $I_{\mathrm{p}, \mathrm{a}} / I_{\mathrm{p}, \mathrm{c}}$ is equal to 1 . This is typical for irreversibly adsorbed organic substances with reversible charge transfer kinetics [22-27]. It also means that the energy of both the reactant and the product is similar. Because desorption at $\mathrm{pH} 4.5$ is quite slow, we can neglect the small fraction of molecules that will dissolve and diffuse from the electrode surface. So the electrode process corresponds only to the charge transfer kinetics of the irreversibly chemisorbed adriamycin.

Glassy carbon is an isotropic material and so all the active sites on the electrode surface can be regarded as equivalent. Based on the results in Fig. 1, Langmuir isotherm behaviour can be considered for the formation of a sub-monolayer of adsorbed material, with lateral interactions between adsorbed species being negligible; no processes involving solution-resident species can occur as there is no adriamycin in solution. For $v$ lower than $10 \mathrm{mV} \mathrm{s}^{-1}$, the equation:

$\Gamma_{\text {adr }}=4 R T l_{\mathrm{p}, \mathrm{a}} / n^{2} F^{2} A v$

enables the calculation of the total surface concentration of adriamycin, $\Gamma_{\mathrm{adr}}$, adsorbed onto the glassy carbon surface [22-27].

The determination of the total surface concentration of adriamycin, $\Gamma_{\text {adr }}$, adsorbed onto a GCE surface that was immersed in a $50 \mathrm{nM}$ adriamycin solution for $3 \mathrm{~min}$, was done using Eq. 1, and the data presented in Table 2. Substituting the number of electrons transferred $n=2$, the surface area of the GCE $A=0.9 \mathrm{~cm}^{2}$ and the scan 

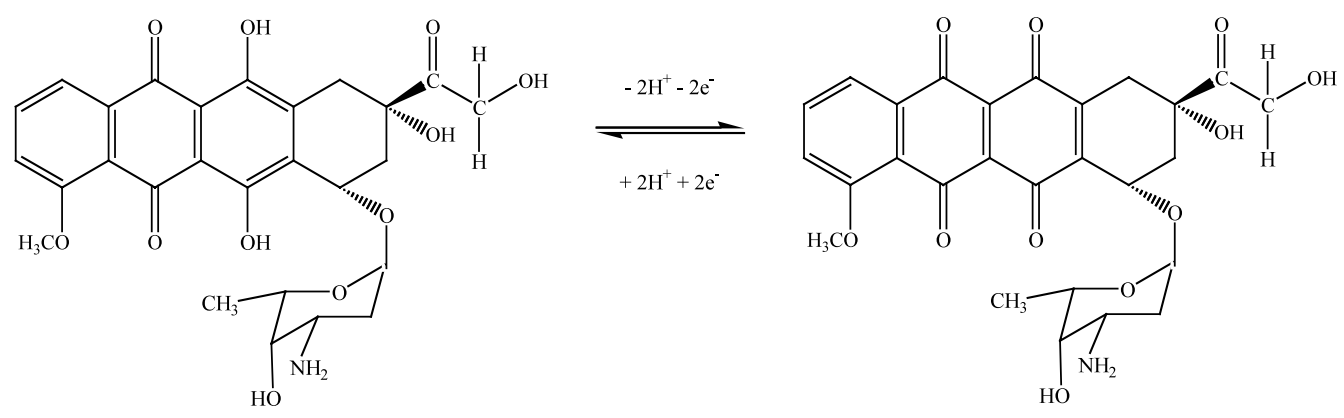

Scheme 2. Mechanism for the oxidation of adriamycin.

rate $v=1 \mathrm{mV} \mathrm{s}^{-1}$, a total surface concentration of adriamycin of $\Gamma_{\mathrm{adr}}=2.57 \times 10^{-12} \mathrm{~mol} \mathrm{~cm}^{-2}$, or $1.5 \times$ $10^{12}$ molecules per $\mathrm{cm}^{2}$, is obtained.

\subsection{Atomic Force Microscopy characterisation of adsorbed adriamycin}

The glassy carbon surface is rough and has high irregularities. For AFM studies, it is necessary to have an atomically flat substrate to resolve the molecular adsorbed layer clearly so that the characterisation of adriamycin adsorption by MAC mode AFM imaging was performed using HOPG. The HOPG electrode presents a root mean square (RMS) roughness of less than $0.06 \mathrm{~nm}$ compared with the GCE with a RMS roughness of $2.10 \mathrm{~nm}$, as calculated from typical $1000 \times$ $1000 \mathrm{~nm}$ scan size contact AFM images in air of HOPG and glassy carbon, respectively. MAC mode AFM is a technique that allows the visualisation of the molecules that are attached at the electrode surface by soft interactions. The adsorption of adriamycin molecules at the HOPG surface proceeded very fast and MAC mode AFM images confirmed the capacity of adriamycin molecules to adsorb spontaneously.

Adriamycin molecules adsorb freely at the HOPG surface after immersing the HOPG for fixed times into solutions of different concentrations of adriamycin, $1 \mathrm{nM}, 50 \mathrm{nM}$ and $1 \mu \mathrm{M}$, in $\mathrm{pH} 4.50 .1 \mathrm{M}$ acetate buffer electrolyte. The in situ MAC mode AFM imaging of adriamycin adsorbed onto the HOPG surface, like the voltammetric experiments in glassy carbon, was performed in $\mathrm{pH} 4.50 .1 \mathrm{M}$ acetate buffer electrolyte solution. Adriamycin films formed on HOPG were very stable in time, in agreement with the electrochemical results previously obtained with the GCE.

After $30 \mathrm{~s}$ free adsorption from a $1 \mathrm{nM}$ adriamycin solution, the adriamycin molecules preferentially attached near the step edges of the HOPG surface. Aggregates with dimensions between 70 and $120 \mathrm{~nm}$ diameter and approximately $0.7 \mathrm{~nm}$ height were observed (Fig. 9(A)). Considering the dimensions of an adriamycin molecule with a critical volume of 1368.50
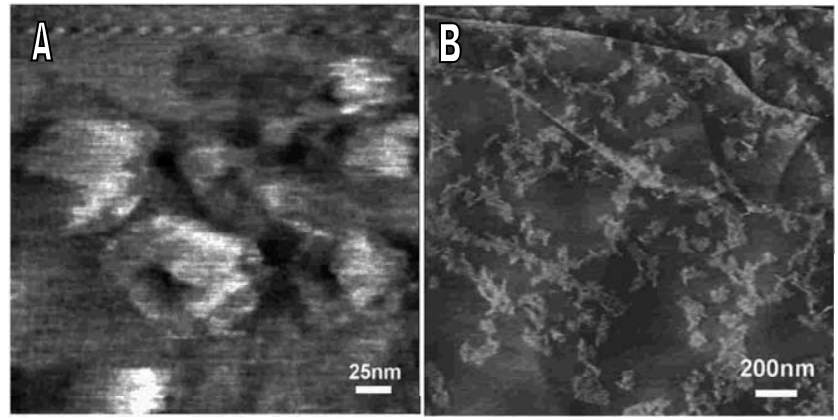

Fig. 9. MAC mode AFM images obtained in $\mathrm{pH} 4.50 .1 \mathrm{M}$ acetate buffer electrolyte of adriamycin adsorbed onto HOPG, after free adsorption from a pH $4.50 .1 \mathrm{M}$ acetate buffer electrolyte $1 \mathrm{nM}$ adriamycin solution: (A) $30 \mathrm{~s}$ and (B) $3 \mathrm{~min}$.

$\mathrm{cm}^{3} \mathrm{~mol}^{-1}$, these aggregates correspond to a monolayer of close-packed adriamycin molecules. When the HOPG is left for $3 \mathrm{~min}$ in the same solution, the adriamycin aggregates grow and cover the HOPG more uniformly (Fig. 9(B)).

A two-dimensional topographic image with corresponding three-dimensional representation, Fig. 10(A and $\mathrm{B}$ ), shows that the adriamycin film formed onto HOPG after 3 min free adsorption from a $50 \mathrm{nM}$ adriamycin solution has a broken aspect. The height of the film, $0.68 \pm 0.1 \mathrm{~nm}$ is similar to the height of the adriamycin aggregates formed at lower concentration, which confirms that the aggregates have grown to form a monolayer.

Differential pulse voltammetric results (Fig. 1) demonstrated that when a $1 \mu \mathrm{M}$ concentration of the adriamycin in solution was used the surface of the GCE was saturated with adriamycin molecules. After $3 \mathrm{~min}$ free adsorption from $1 \mu \mathrm{M}$ adriamycin solution, the AFM images showed a complete coverage of the HOPG surface (Fig. 11(A, B and C)).

The images revealed large, flat terraces of several hundred nanometers width, sometimes larger than $1 \mu \mathrm{m}$ and the thickness of the adriamycin film was $0.7 \pm 0.1$ $\mathrm{nm}$ as measured in by section analysis inside the images (Fig. 11(D)). 

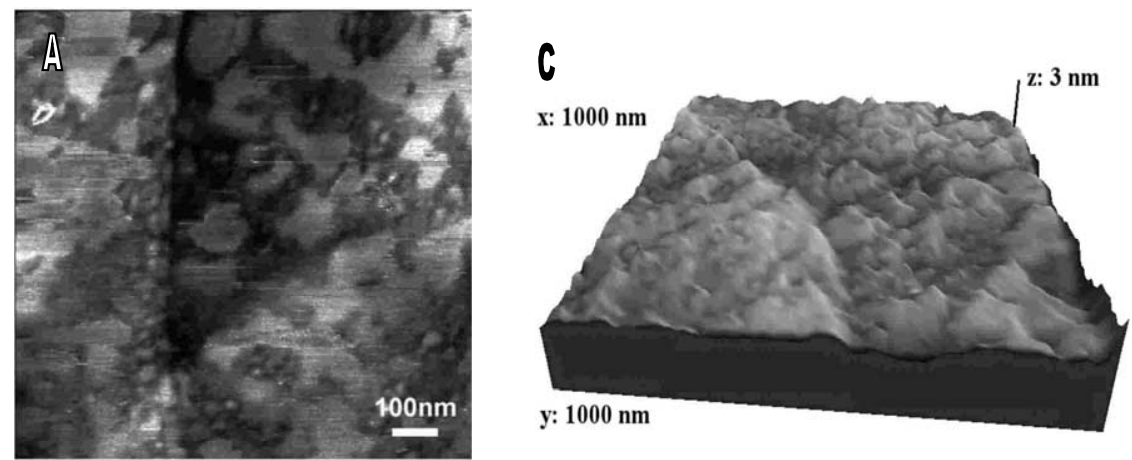

Fig. 10. (A) MAC mode AFM image obtained in $\mathrm{pH} 4.50 .1 \mathrm{M}$ acetate buffer electrolyte of adriamycin adsorbed onto HOPG, after 3 min free adsorption from a pH 4.5 0.1 M acetate buffer electrolyte $+50 \mu \mathrm{M}$ adriamycin solution; (B) Three-dimensional processing of image (A).

The thickness of the monolayer calculated from the AFM images is equal to the expected dimensions of the adriamycin molecule perpendicular to the aromatic rings of approximately $0.8 \mathrm{~nm}$. This leads to the conclusion that the adriamycin molecules have the planar aromatic rings lying flat on the surface (Scheme 2), bonding by hydrophobic interaction with the hydrophobic carbon surface. The side groups of the adriamycin molecules can also form additional hydrogen bonds to the surface. However, in MAC mode AFM images obtained for the adriamycin layer adsorbed at this high concentration, differences in the film height of $1.55 \pm$ $0.15 \mathrm{~nm}$ could be also observed sporadically, which suggest the initiation of the formation of a second layer of adsorbed adriamycin molecules.

\subsection{Electroanalytical determinations}

The fact that adriamycin chemisorbs strongly and irreversibly on the electrode surface maintaining its electroactive behaviour, enables the voltammetric detection of unusually low concentrations of adriamycin.

A differential pulse voltammogram (Fig. 12 curve a), was obtained for $1 \times 10^{-10} \mathrm{M}$ adriamycin in $\mathrm{pH} 4.50 .1$ $\mathrm{M}$ acetate buffer electrolyte solution. Differential pulse voltammograms for standard additions of adriamycin solutions corresponding to bulk concentrations between 0.1 and $1 \mathrm{nM}$ in $\mathrm{pH} 4.50 .1 \mathrm{M}$ acetate buffer electrolyte solutions, are shown in Fig. 12(a). Good linearity was found between peak current and concentration described by the equation $I_{\mathrm{p}, \mathrm{a}} / \mathrm{A}=3.20 \times 10^{-9}+18.95$
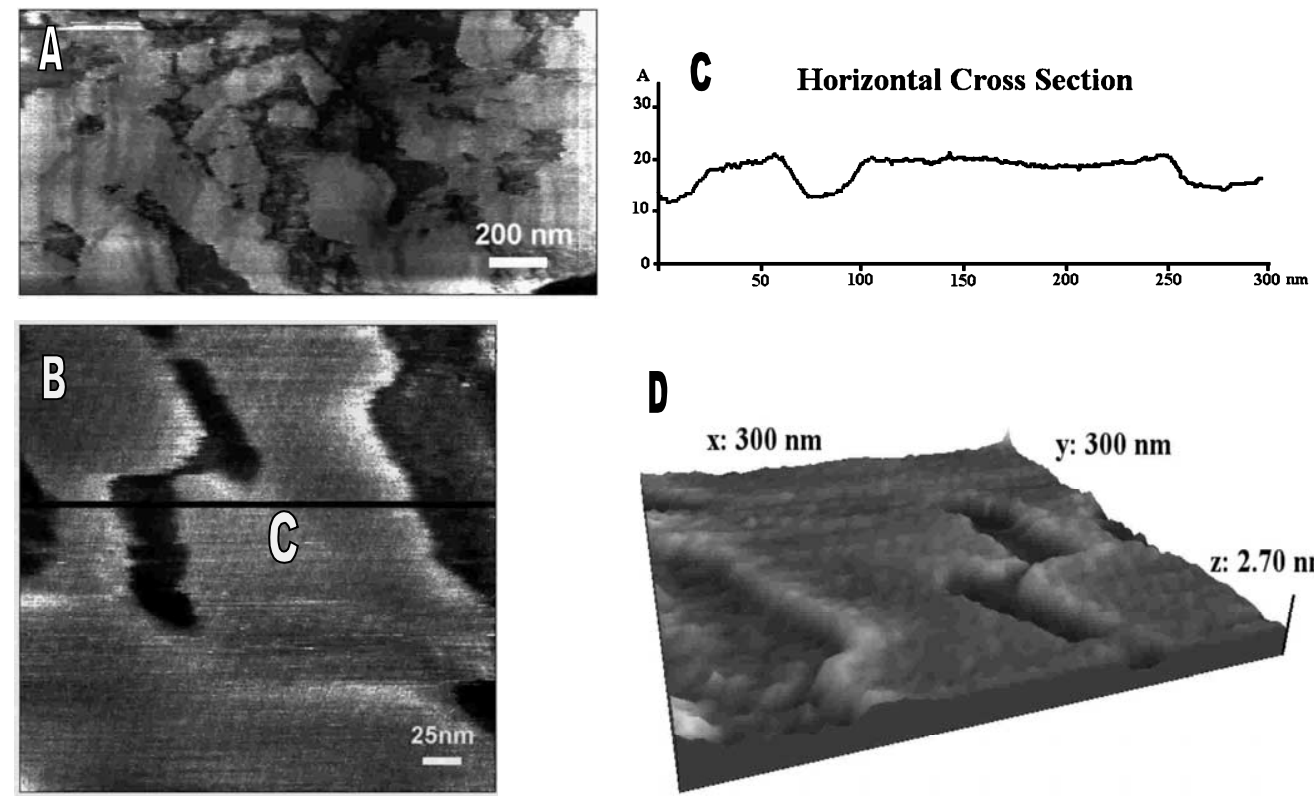

D

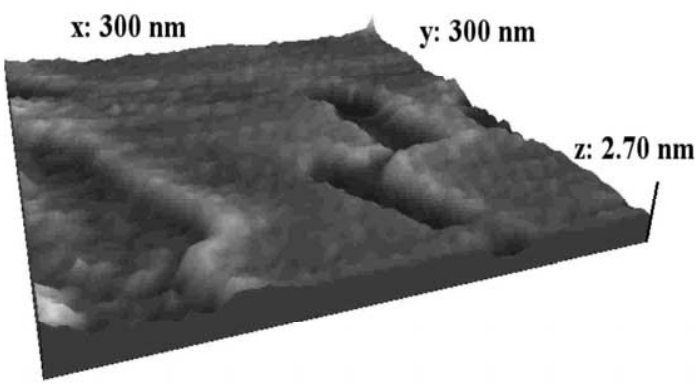

Fig. 11. (A), (B) MAC mode AFM obtained in pH $4.50 .1 \mathrm{M}$ acetate buffer electrolyte of adriamycin adsorbed onto HOPG, after 3 min free adsorption from a pH 4.5 0.1 M acetate buffer electrolyte $1 \mu \mathrm{M}$ adriamycin solution; (C) Three-dimensional processing of the image (B); (D) Crosssection inside image (B). 

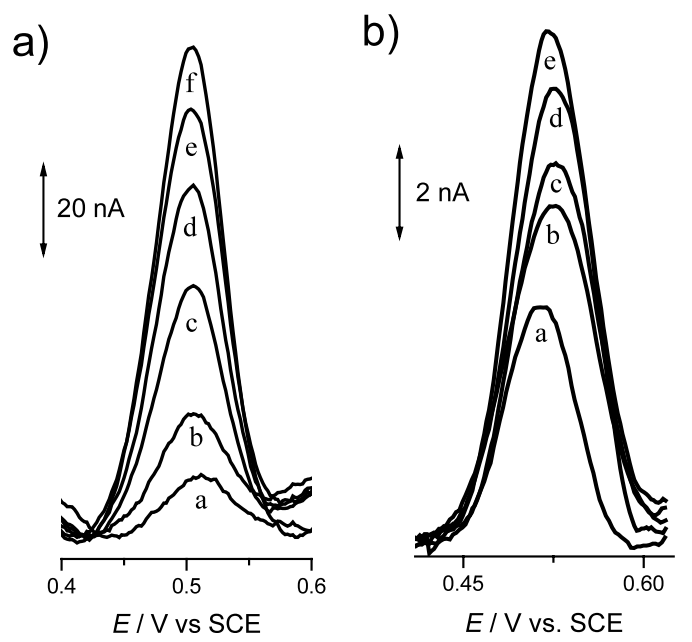

Fig. 12. Standard addition background-subtracted differential pulse voltammograms obtained in $\mathrm{pH} 4.50 .1 \mathrm{M}$ acetate buffer electrolyte with GCE: (a) adriamycin solutions: (a) $1 \times 10^{-10}$, (b) $3 \times 10^{-10}$, (c) $5 \times 10^{-10}$, (d) $7 \times 10^{-10}$, (e) $8 \times 10^{-10}$, (f) $1 \times 10^{-9} \mathrm{M}$; (b) adriamycin adsorbed onto GCE surface after deposition of $3 \mathrm{~min}$ from $\mathrm{pH} 4.5$ $0.1 \mathrm{M}$ acetate buffer electrolyte adriamycin solutions: (a) $1 \times 10^{-10}$, (b) $3 \times 10^{-10}$, (c) $5 \times 10^{-10}$, (d) $9 \times 10^{-10}$, (e) $1 \times 10^{-9} \mathrm{M}$.

[Adr] $/ \mathrm{M}\left(R=0.997, n=10\right.$, S.D. $\left.=4.98 \times 10^{-10}\right)$. This allowed attaining a detection limit of $7.88 \times 10^{-11} \mathrm{M}$ to be attained, based on three times the noise level [29].

After recording the differential pulse voltammograms at each concentration of adriamycin in solution, the glassy carbon with adsorbed adriamycin was transferred to buffer electrolyte solution, being first thoroughly washed with a jet of deionised water. The peak currents obtained by differential pulse voltammetry for adsorbed adriamycin, Fig. 12(b), showed good linearity, described by the equation $I_{\mathrm{p}, \mathrm{a}} / \mathrm{A}=5.13 \times 10^{-9}+5.53[\mathrm{Adr}] / \mathrm{M}$ $\left(R=0.946, n=10\right.$, S.D. $\left.=6.08 \times 10^{-10}\right)$, and a detection limit of $3.30 \times 10^{-10} \mathrm{M}$.

The strong and irreversible adsorption of adriamycin onto GCE, allowed detection limits 10000 times lower than the usual limits for voltammetric methods for most organic compounds. The detection limit of the order of $10^{-11} \mathrm{M}$ presented in this paper for adriamycin is the lowest reported in the literature for this substance using voltammetric methods.

\section{Conclusions}

The detection limit of the order of $10^{-11} \mathrm{M}$ for adriamycin confirms the valuable use of voltammetry to detect trace amounts of strongly adsorbing electroactive drugs in biological fluids. The development of voltammetric biosensor based devices is foreseen to study the mechanism of interaction and interfacial phenomena between drugs, such as adriamycin, and crucial biological targets as membranes, proteins, and nucleic acids.
The spontaneous adsorption of adriamycin molecules at carbon surfaces was demonstrated by electrochemistry at glassy carbon and by in situ MAC mode AFM images on HOPG surfaces, both obtained in acetate buffer electrolyte. The monolayer films obtained were stable with time, covering almost the entire surface, which suggests a strong interaction between the adriamycin molecules and the carbon surfaces via hydrophobic interaction and hydrogen bonding.

\section{Acknowledgements}

Financial support from Fundação para a Ciência e Tecnologia (FCT) project POCTI (co-financed by the European Community fund FEDER), ICEMS (Research Unit 103) and European Projects ERB-ICT15CT960804 and QLK3-2000-01311, are gratefully acknowledged.

\section{References}

[1] J.W. Lown, Chem. Soc. Rev. (1993) 165.

[2] A. Di Marco, M. Gaetani, B.M. Scarpinato, Cancer Chemother. Rep. 63 (1969) 33.

[3] S.Y. Zhou, A. Starkov, M.K. Froberg, R.L. Leino, K.B. Wallace, Cancer Res. 61 (2001) 771.

[4] G. Minotti, G. Cairo, E. Monti, FASEB J. 13 (1999) 199.

[5] E.L. Kostoryz, D.M. Yourtee, Mutat. Res. 490 (2001) 131.

[6] K. Kiyomiya, S. Matsuo, M. Kuruebe, Cancer Chemother. Pharmacol. 47 (2001) 51.

[7] H. Berg, G. Horn, U. Luthardt, Bioelectrochem. Bioenerg. 8 (1981) 537.

[8] C.A. Frederick, L.D. Williams, G. Ughetto, G.A. Van Der Marel, J.H. Van Boom, A. Rich, A.H.J. Wang, Biochemistry 29 (1990) 2538 .

[9] L.A. Lipscomb, M.E. Peek, F.X. Zhou, J.A. Bertrand, D. Vanderveer, L.D. Williams, Biochemistry 33 (1994) 3649.

[10] F. Zunino, R. Gambetta, A. Di Marco, A. Velcich, A. Zaccara, F. Quadrifoglio, V. Crescenzi, Biochim. Biophys. Acta 476 (1977) 38.

[11] K. Kiyomiya, S. Matsuo, M. Kuruebe, Cancer Res. 61 (2001) 2467.

[12] A.M. Oliveira-Brett, S.H.P. Serrano, J.A.P. Piedade, in: R.G. Compton, G. Hancock (Eds.), Comprehensive Chemical Kinetics, vol. 37 (Chapter 3), Elsevier, Amsterdam, 1999, p. 91.

[13] A.M. Oliveira Brett, S.H.P. Serrano, T.A. Macedo, D. Raimundo, M.H. Marques, M.A. La-Scalea, Electroanalysis 7 (1996) 992.

[14] A.M. Oliveira Brett, M. Vivan, I.R. Fernandes, J.A.P. Piedade, Talanta 56 (2002) 959.

[15] A.M. Oliveira-Brett, J.A.P. Piedade, S.H.P. Serrano, Electroanalysis 12 (2000) 969.

[16] L.A. Sternson, G. Thomas, Anal. Lett. 10 (1977) 99.

[17] J. Hu, Q. Li, Anal. Sci. 15 (1999) 1215.

[18] E.N. Chaney, R.P. Baldwin, Anal. Chem. 54 (1982) 2556.

[19] R.P. Baldwin, D. Packett, T.M. Woodcock, Anal. Chem. 53 (1981) 540.

[20] W. Han, S.M. Lindsay, T.W. Jing, Appl. Phys. Lett. 69 (1996) 4111. 
[21] J.G. Osteryoung, J.J. O’Dea, in: A.J. Bard (Ed.), Electroanalytical Chemistry, vol. 14, Marcel Dekker, New York, 1986, p. 53.

[22] E. Laviron, in: A.J. Bard (Ed.), Electroanalytical Chemistry, vol. 12, Marcel Dekker, New York, 1982, p. 53.

[23] C.M.A. Brett, A.M. Oliveira Brett, Electrochemistry, Principles, Methods, and Applications, Oxford University Press, Oxford, 1993.

[24] A.P. Brown, F.C. Anson, Anal. Chem. 49 (1977) 1589.
[25] A.T. Hubbard, F.C. Anson, in: A.J. Bard (Ed.), Electroanalytical Chemistry, vol. 4, Marcel Dekker, New York, 1970, p. 129.

[26] R.W. Murray, Acc. Chem. Res. 13 (1980) 135.

[27] R.W. Murray, in: A.J. Bard (Ed.), Electroanalytical Chemistry, vol. 13, Marcel Dekker, New York, 1981, p. 191.

[28] N. Oyama, F. Anson, J. Electrochem. Soc. 127 (1980) 640.

[29] C.M.A. Brett, A.M. Oliveira Brett, Electroanalysis, Oxford University Press, Oxford, 1998 